CORRECTIONS

\title{
Bones of the hand I
}

The authors of this Endgames Anatomy quiz ( $B M J$ 2012;344:e505, doi:10.1136/bmj.e505) would like to make a correction to their labelling of the left hand. Answers B and C should have been reversed, as on this radiograph Answer B more accurately identifies the "triquetral" and Answer C the "pisiform."

Cite this as: BMJ 2012;345:e6113

๑ BMJ Publishing Group Ltd 2012 